\title{
Nonequilibrium transitions in complex networks: A model of social interaction
}

\author{
Konstantin Klemm, ${ }^{*}$ Víctor M. Eguíluz, ${ }^{\dagger}$ Raúl Toral, ${ }^{*}$ and Maxi San Miguel ${ }^{\S}$ \\ Instituto Mediterráneo de Estudios Avanzados IMEDEA (CSIC-UIB), E07122 Palma de Mallorca, Spain
}

(Received 1 November 2002; published 26 February 2003)

\begin{abstract}
We analyze the nonequilibrium order-disorder transition of Axelrod's model of social interaction in several complex networks. In a small-world network, we find a transition between an ordered homogeneous state and a disordered state. The transition point is shifted by the degree of spatial disorder of the underlying network, the network disorder favoring ordered configurations. In random scale-free networks the transition is only observed for finite size systems, showing system size scaling, while in the thermodynamic limit only ordered configurations are always obtained. Thus, in the thermodynamic limit the transition disappears. However, in structured scale-free networks, the phase transition between an ordered and a disordered phase is restored.
\end{abstract}

DOI: 10.1103/PhysRevE.67.026120

PACS number(s): 89.75.Fb, 05.50.+q, 87.23.Ge

\section{INTRODUCTION}

Lattice models are a powerful basic instrument in the study of phase transitions in equilibrium statistical mechanics, as well as in nonequilibrium systems [1]. Traditionally, equilibrium phase transitions have been studied in regular lattices, with the critical temperature being a nonuniversal quantity that depends on the particular lattice under consideration, while critical exponents and some amplitude ratios are universal quantities depending only on spatial dimension and some symmetries of the order parameter. The detailed structure of the regular network connections is, in most cases, irrelevant in the sense of the renormalization group. However, recent research in the structure and topology of complex networks $[2,3]$ has shown that social interactions and, more generally, biological and technological networks, are far from being regular as well as being also far from a random network or from a mean-field network linking all to all. This has triggered the study of standard models of statistical mechanics in these complex networks. In particular, recent studies of the Ising model in the so-called small world [4] and the scale-free [5] networks have shown that the behavior of the model differs from that observed in a regular network.

The statistical properties of real-world social networks vary strongly. The degree distribution can be single-scale, broad-scale or scale-free [6,7]. See also three recent studies on networks of individual electronic mail communication [8-10]. Due to the lack of a single model encompassing the topological features of social networks, we consider a few established network models aiming to unveil the effect of different aspects of the topology.

A small-world network [4] is generated by rewiring with a probability $p$ the links of a regular lattice by long-distance random links. The presence of a small fraction of "short

\footnotetext{
*Electronic address: klemm@nbi.dk

†Electronic address: victor@imedea.uib.es; URL: http:// www.imedea.uib.es/ victor

${ }^{\ddagger}$ Electronic address: raul@imedea.uib.es

${ }^{\S}$ Electronic address: maxi@imedea.uib.es
}

cuts" connecting otherwise distant points, drastically reduces the average shortest distance between any pair of nodes in network, keeping the clustering high. The small-world networks generated by rewiring links have degree distributions with exponential tails. In contrast, scale-free networks [5] are characterized by a fat-tailed (power law) degree distribution. They are usually modelled by growing networks and preferential attachment of links.

The Ising model in small-world topologies shows a change of behavior from the regular case to the mean-field characteristics. In Ref. [11] it is shown analytically that for a small world lattice, obtained from rewiring with probability $p$ the links of a (one-dimensional) 1D ring lattice with $2 k$ nearest neighbors interactions, the crossover temperature to the mean-field critical behavior varies for $p \ll 1$ as $T_{c o}(p) \propto$ $-k(k+1) / \ln (p)$, whereas the critical temperature scales as $T_{c}(p) \propto-2 k / \ln (p)$, so that a ferromagnetic ordered phase exists for any finite value of $p$. The crossover to mean-field behavior in small-world ring lattices has been further discussed in Refs. [12,13], whereas numerical results in 2D and 3D lattices have been reported in Ref. [14]. Interestingly, if directed links are considered, not only the critical temperature changes but the nature of the transition also switches from second order to first order [15].

A much different behavior is observed in scale-free networks. This can be related to the influence of the presence of so-called hubs, i.e., units whose degree is much larger than average. This is well illustrated by the behavior of the Ising model in scale-free networks with degree distribution $P(k)$ $\propto k^{-\gamma}, \gamma>1$. The results of Refs. [16,17] show that equilibrium systems exhibit a phase diagram that is qualitatively different from the mean-field case. In particular, the Ising model in a random scale-free networks shows an infinite critical temperature in the thermodynamic limit of an infinite number of nodes. In fact, an analytical theory has been developed connecting the exponent of the degree distribution and the critical behavior of the Ising model [18-20].

In this paper, we address the question of the role played by the topology of complex networks in nonequilibrium transitions of models in which there is interaction between the variables associated with the nodes connected by links in the network. This is a natural next step beyond the analysis of 
equilibrium, Ising-type models in these complex networks. Simple nonequilibrium models closely related to percolation have been already considered [21-23]. Here, and given the social motivation and relevance of these complex networks, we have chosen to analyze the model proposed by Axelrod for the dissemination of culture [24]. The spreading process in this model cannot be reduced to a percolation process. The model rather describes a competition between dominance and spatial coexistence of different states in a nonequilibrium dynamics of coupled Potts-type models. The model was originally considered by Axelrod in a square lattice. The statistical mechanics analysis of the model in this regular twodimensional network identifies a nonequilibrium orderdisorder phase transition [25]. However, it is interesting to notice that, in his original paper, Axelrod already discussed the relevance of the topology, speculating that "with random long-distance interactions, the heterogeneity sustained by local interaction cannot be sustained." In particular, we consider here this question.

In the following section, we introduce the original model proposed in Ref. [24] and summarize briefly the main results in regular 2D networks. The model in small-world and scalefree networks is presented in Secs. III and IV, respectively. The nonequilibrium transition is shown to disappear in the thermodynamic limit of a scale-free network. We then consider in Sec. V, a structured scale-free network $[26,27]$ which shares characteristics of small-world and scale-free networks. A nonequilibrium transition is shown to persist for large systems in this network. Our conclusions are summarized in Sec. VI.

\section{THE MODEL}

The model we study is defined [24] by considering $N$ agents as the sites of a network. The state of agent $i$ is a vector of $F$ components (cultural features) $\left(\sigma_{i 1}, \sigma_{i 2}, \ldots, \sigma_{i F}\right)$. Each $\sigma_{i f}$ can take any of the $q$ integer values (cultural traits) $1, \ldots, q$, initially assigned independently and with equal probability $1 / q$. The time-discrete dynamics is defined as iterating the following steps:

(1) Select at random a pair of sites of the network connected by a bond $(i, j)$.

(2) Calculate the overlap (number of shared features) $l(i, j)=\sum_{f=1}^{F} \delta_{\sigma_{i f}, \sigma_{j f}}$.

(3) If $0<l(i, j)<F$, the bond is said to be active and sites $i$ and $j$ interact with probability $l(i, j) / F$. In case of interaction, choose $g$ randomly such that $\sigma_{i g} \neq \sigma_{j g}$ and set $\sigma_{i g}$ $=\sigma_{j g}$.

In any finite network the dynamics settles into an absorbing state, characterized by the absence of active bonds. Obviously all the $q^{F}$ completely homogeneous configurations are absorbing. Homogeneous means here that all the sites have the same value of the cultural trait for each cultural feature. Inhomogeneous states consisting of two or more homogeneous domains interconnected by bonds with zero overlap are absorbing as well. A domain is a set of contiguous sites with identical cultural traits. We define an order parameter in this system $[25,28]$ as the relative size of the largest

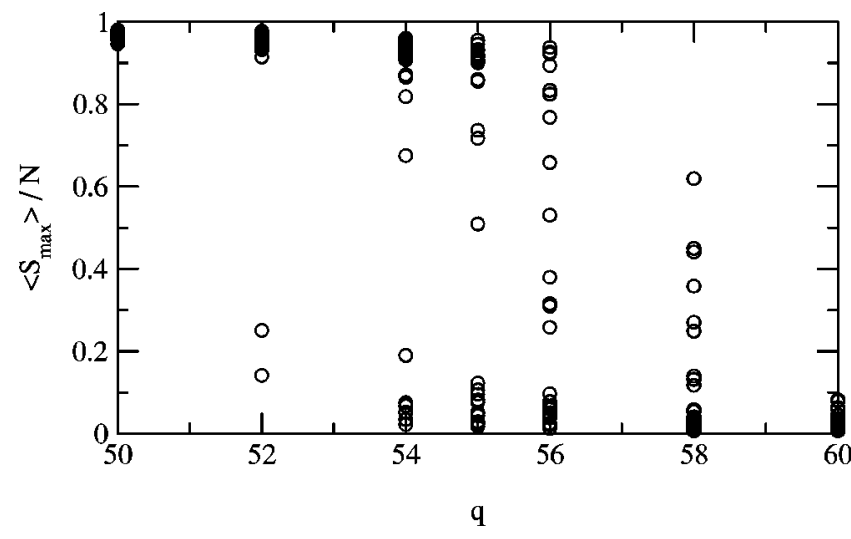

FIG. 1. The order parameter $S_{\text {max }} / N$ as a function of $q$ in regular lattices with $N=100^{2}$ nodes for $F=10$. For each value of $q$ the outcome of 32 independent realizations is shown. The transition occurs for $q \approx 55$ (see Fig. 2).

homogeneous domain $S_{\max } / N$, being $N$ the number of sites in the network.

Previous results have been obtained in square lattices with nearest neighbor interaction. A variation of the model with initial distribution of traits according to a Poisson rather than a uniform distribution shows a nonequilibrium order-disorder phase transition where the number of traits $q$ plays the role of a control parameter [25]. The system reaches ordered absorbing states for $q<q_{c}\left[S_{\max }=\mathcal{O}(N)\right]$ and disordered states for $q>q_{c}\left(S_{\max } \ll N\right)$. The same type of phase transition occurs in the original model with a uniform initial distribution of traits [28].

When comparing the effect of complex networks in this phase transition with the equilibrium Ising transition one should notice several conceptual differences. First, this is a sort of zero-temperature transition in which ordered or disordered states exists with no reference to thermal fluctuations. In fact, the effect of small noise in this system is essential, revealing the presence of metastable states and changing the phase diagram in a nontrivial way [28]. A second related point is that the control parameter of the transition $q$, is here not a collective property of the system as temperature, but rather an ingredient of the definition of the system itself. In a way, the transition occurs going from one system to another as $q$ is changed. Finally, and in reference to critical properties and exponents, we note that the transition (except for $F=2$ ) is of first-order type. In Fig. 1, we plot the final values for the order parameter, obtained for 32 different realizations of the dynamics. Notice that for $q$ $<50$ all the systems end up in a homogenous state that basically fills up the entire lattice $\left(S_{\max } / N \approx 1\right)$, whereas for $q>60$ the maximum homogenous regions obtained are very small. This is the order-disorder phase transition discussed before. For $50<q<60$, we observe bistability in the sense that the system settles around any of two mean values for the order parameter. This bistability, which is usually associated with first-order phase transitions, is clearly made explicit in the corresponding histogram shown in Fig. 2 where the two preferred values appear as maxima of the histogram. The 


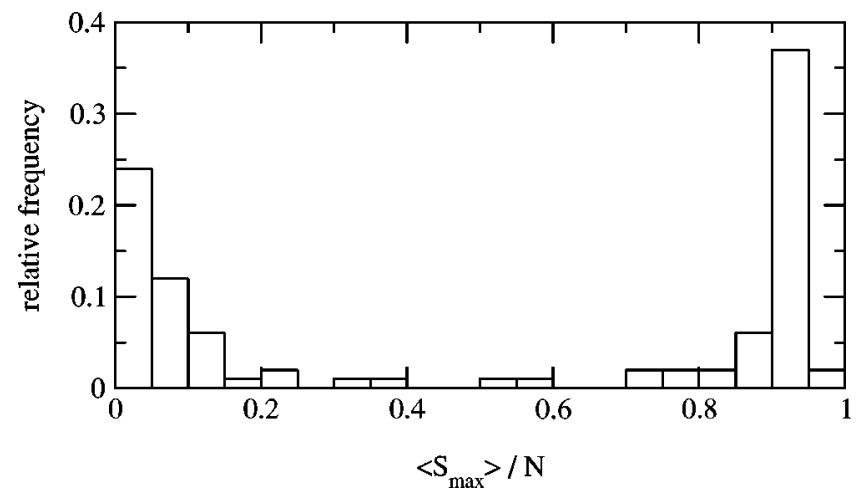

FIG. 2. Distribution of the order parameter at $q=55, F=10$ in a square lattice of size $N=100^{2}$. The distribution is based on 100 independent realizations.

transition point corresponds to $q=q_{c}$ for which these two values are equally probable.

\section{SMALL-WORLD NETWORK}

Social networks are far from being regular or completely random. However, they also share some features with them. On the one hand, social networks are known to be small [30], i.e., any pair of nodes in the network can be connected following a number of links much smaller than the size of the network. This is also observed in random networks, where the average shortest distance between pair of nodes (the socalled path length $\ell$ ) increases logarithmically with the size of the network $\ell \sim \ln N$, while in regular lattice in $d$ dimensions $\ell \sim N^{1 / d}$. On the other hand, social networks are also known to form cliques [31], i.e., groups of nodes highly connected between them. "Cliquishness" can be characterized by the so-called clustering coefficient $C$, which is defined as the relative number of closed triangles in the network. Regular lattices can show large clustering, while in random networks $C \sim N^{-1}$. High clustering and short path length define a small-world network.

The first model encompassing the small-world effect was introduced in Ref. [4] proposing an algorithm that interpolates between a random and a regular lattice. First one generates a two-dimensional regular lattice with bonds between nearest neighbors and open boundary conditions. Then for each bond $(i j)$, with probability $p$ detach the bond from node $j$ and attach it to a node $l$ instead. Node $l$ is chosen at random with the restriction that duplicate and selfconnections are excluded. The parameter $p$ interpolates between the original regular lattice ( $p=0$, no rewiring) and a network very similar to a random graph $(p=1)$. Thus, in the limiting case $p=0$, we have a network with high clustering but also large path length; in the limit $p=1$, we have networks with small path length but also small clustering. For intermediate values of $p$ the algorithm generates networks with high clustering and small path length.

We now study the behavior of Axelrod's model in dependence of $p$. A small-world network is used from the beginning of each simulation run. Figure 3 shows the dependence of the order parameter on $q$, for three different values of $p$.

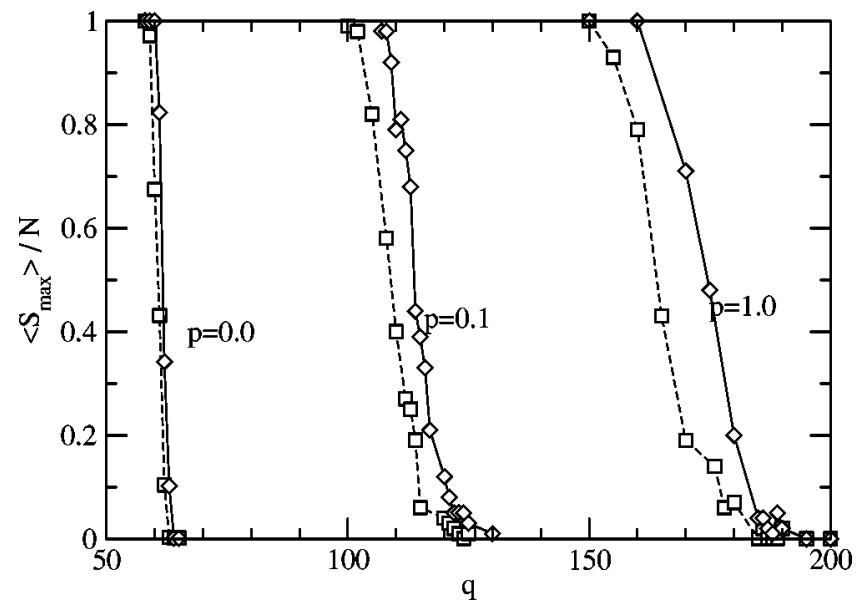

FIG. 3. The average order parameter $\left\langle S_{\max }\right\rangle / N$ as a function of $q$ for three different values of the small-world parameter $p$. System sizes are $N=500^{2}$ (squares) and $N=1000^{2}$ (diamonds); number of features $F=10$. Each plotted value is an average over 100 runs with independent rewiring $(p>0)$ and independent initial conditions.

For any fixed value $p>0$, we find a nonequilibrium phase transition which becomes sharp and well defined as the system size increases. There is a critical value $q_{c}$ of the control parameter which separates the ordered and the disordered state, just as in regular lattices. However, $q_{c}$ increases with the amount of spatial disorder. This is clearly shown in the $(p, q)$-phase diagram, Fig. 4. The filled area above the $\left[p, q_{c}(p)\right]$ curve represents the disordered states, the area below the curve represents the ordered states. Consequently,

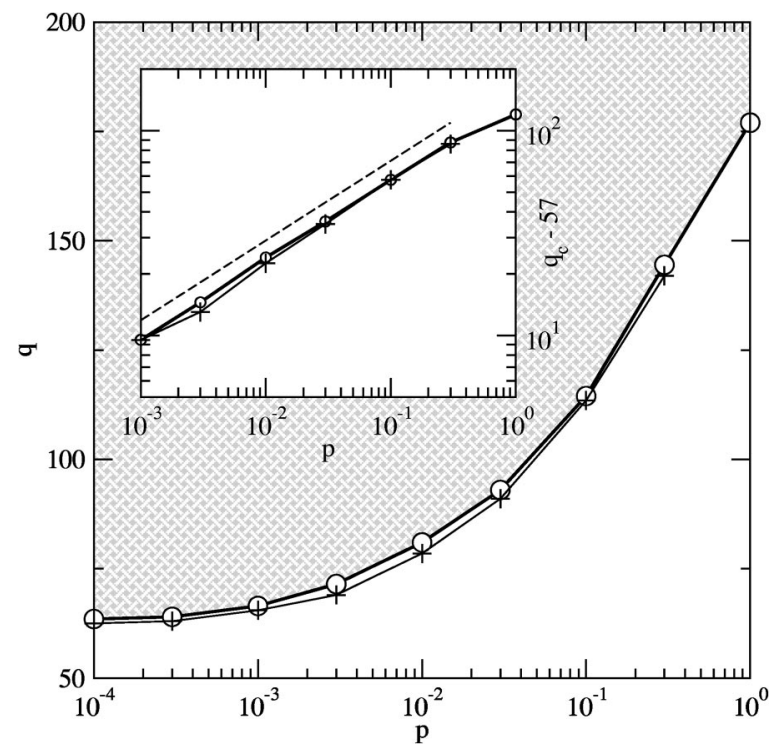

FIG. 4. Phase diagram for the Axelrod model in a small-world network. The curve separates parameter values $(p, q)$ which produce a disordered state (shaded area) from those with ordered outcome (white area). For a given $p$ the plotted value $q_{c}$ is the one for which the value of the order parameter is closest to the, somewhat arbitrary but small, value 0.1 for system size $N=500^{2}$ and $F=10$. Inset: After subtraction of a bias $q_{c}(p=0)=57, q_{c}(p)$ follows a power law $\propto p^{0.39}$ (dashed line). 


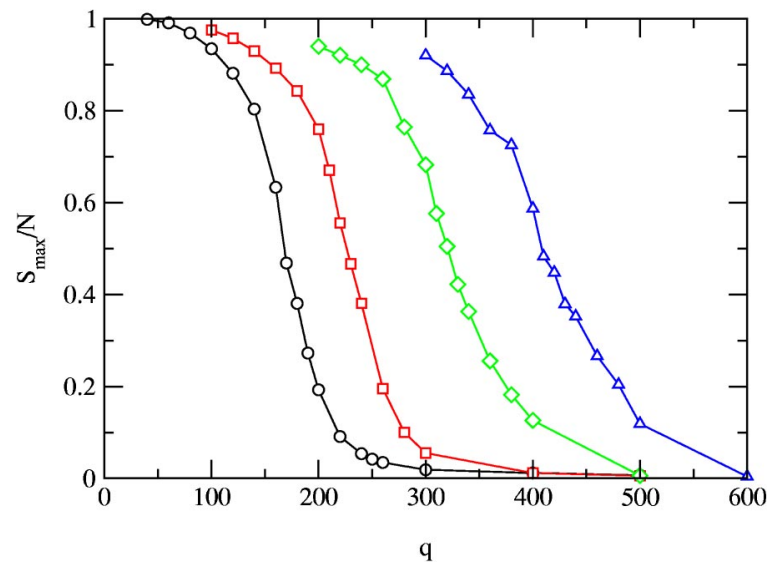

FIG. 5. The average order parameter $\left\langle S_{\max }\right\rangle / N$ in random scalefree networks for $F=10$. Averages are taken over 1000 independent realizations. Different curves are for different system sizes: 1000 (circles), 2000 (squares), 5000 (diamonds), and 10000 (triangles).

for values $q<q_{c}(p)$ the outcome of the dynamics is always complete order, whereas for $q>q_{c}(p)$ only disordered frozen states are encountered. The density $p$ of rewired bonds determines the nature of these frozen states, but for $q<q_{c}(p$ $=1$ ) the system orders by increasing $p$, that is, the number of long-distance links. We find a dependence $q_{c}(p)-q_{c}(p$ $=0) \propto p^{\alpha}$ with $\alpha=0.4$ obtained from a best fit. This result is displayed in the inset of Fig. 4. Therefore, we find the same qualitative result as for the equilibrium Ising model, in the sense that the small-world connectivity favors ordered states.

The robustness of the phase diagram is shown by performing a different dynamical scenario. First, a run of the dynamics in a regular lattice is performed. Only after an absorbing configuration has been reached the lattice is rewired according to the above rewiring procedure with the parameter $p$. After the rewiring, the configuration is not necessarily frozen because the rewiring can introduce active bonds connecting compatible cultures that have been disconnected before. Starting the dynamics again, the system may relax to a different absorbing configuration, which, in general, is more ordered than the configuration reached before the rewiring. After this second phase of relaxation, the order parameter is measured in the absorbing state. We find that the results of this alternative scenario (see Fig. 4) are in good agreement with the ones of the above original scenario, starting with a small-world network in the initial condition.

\section{SCALE-FREE NETWORKS}

One important ingredient missing in the small-world networks considered so far is that the degree distribution does not show a fat tail. Although it is not clear whether social networks present a power law distribution of degree, the evidence indicates that they are ubiquitous in biological and artificial networks [6]. Scale-free networks are characterized by a power law tail in the degree distribution of the form $P(k) \propto k^{-\gamma}$, where the exponent $\gamma$ lies in the range between 2 and 3. Two ingredients have been shown to be sufficient to generate such feature: growing number of nodes and preferential attachment of links.

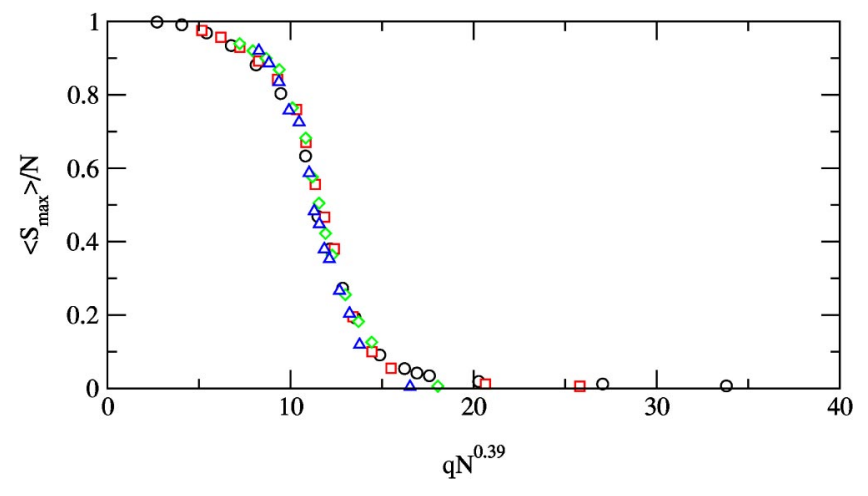

FIG. 6. Rescaled plot of the data shown in Fig. 5 for different system sizes.

The well established Barabási-Albert model is based in these two mechanisms [5]. At each time step a new node is added to the network and attaches $m$ links to an existing node with degree $k$ with probability $\Pi(k) \propto k$. This algorithm generates networks whose degree distribution follows $P(k)$ $=2 m^{2} k^{-3}$, the path length increases as $\ell \sim \ln N$, and the clustering decreases as $C \sim(\ln N)^{2} / N$. We have studied the dynamics of Axelrod's model for the diffusion of culture in scale-free networks following this algorithm. In Fig. 5, we show our results for the order parameter for different system sizes. For a given size $N$, we find a transition at $q_{c}(N)$. We can define the critical value $q_{c}(N)$ as the value where the standard deviation of the distribution of $S_{\max } / N$ reaches the maximum value. We find that $q_{c}(N) \sim N^{0.39}$. Using this result, we observe data collapse with a rescaling $q N^{-\beta}$, see Fig. 6. The best result is obtained for $\beta=0.39$ in excellent agreement with the scaling obtained previously. This indicates that in the thermodynamic limit the transition disappears and the ordered monocultural state establishes in the system. This behavior is similar to the Ising model in regular and scale-free networks: While in a two-dimensional lattice

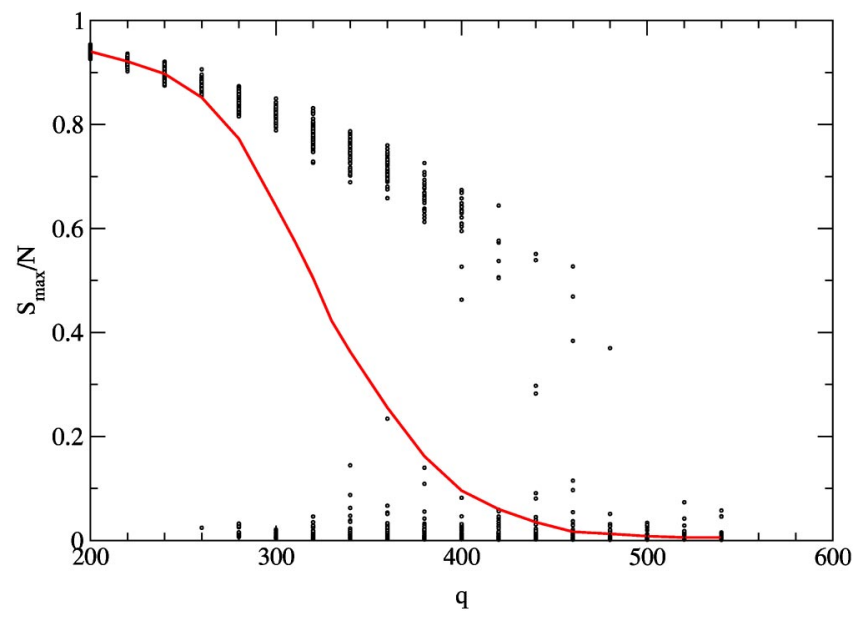

FIG. 7. Order parameter in random scale-free networks of size $N=5000$ with $F=10$. For each value of $q$ the outcomes of 100 independent realizations and the mean value (solid curve) are shown. 


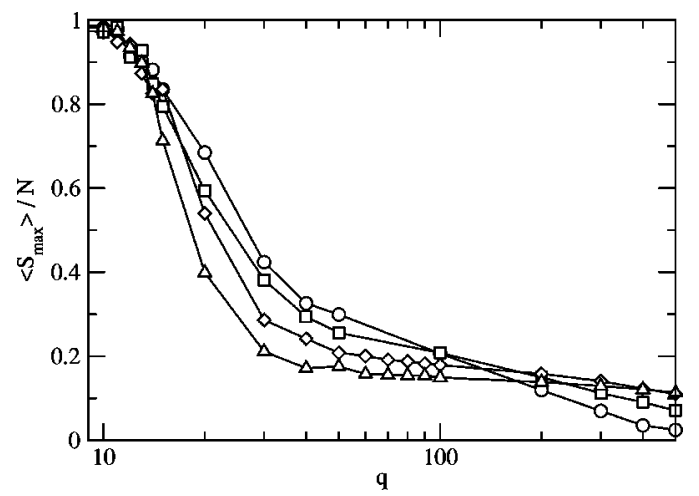

FIG. 8. The average order parameter $\left\langle S_{\max }\right\rangle / N$ as a function of $q$ for $F=10$ in structured scale-free networks. The networks contained $N=1000$ (circles), $N=2000$ (squares), $N=5000$ (diamonds), and $N=10000$ (triangles) nodes with $F=10$ features. Each data point is an average over 32 independent realizations.

the Ising model displays a phase transition at a finite critical temperature, in random scale-free networks an effective transition is observed for finite systems where the effective critical temperature diverges logarithmically with system size. This can be explained by the role of the hubs (nodes with a large number of links) in these networks. They are highly instrumental in establishing ferromagnetic order in the system. The same prominent role is played by the hubs in the case of the dissemination of culture. The hubs help the spreading of cultural traits as can be inferred from the observed dependence with system size. Note, however, that the effective transition of Axelrod's model for a finite system in a scale-free network displays the characteristics of a firstorder transition: We show in Fig. 7 the same type of behavior as observed in Fig. 1 for the regular network. For a range of values of $q$ around $q_{c}$ a realization ends either in an ordered monocultural state or in a disordered frozen configuration, the two preferred values of the order parameter.

\section{STRUCTURED SCALE-FREE NETWORKS}

The scale-free networks considered in the preceding section, underestimate the clustering observed in real networks [32]. A question that merits being addressed is if scale-free networks with high clustering present an absence of the phase transition in the thermodynamic limit. In order to reproduce a high clustering along with a scale-free distribution of the degree, we employ the networks generated by the algorithm proposed in Ref. [26]: Again at each time step we add a new node to the network. The node is attached to the $m$ active nodes in the network. The new node becomes active and one of the $m+1$ active nodes is deactivated with probability $\Pi(k) \propto k^{-1}$. Starting from $m$ fully interconnected active nodes, this algorithm generates scale-free networks with a clustering coefficient $C \approx 5 / 6$ independent of system size. In the following, we call these networks structured scale-free networks because of the large clustering coefficient, the strong negative correlation between degrees of connected nodes [23], and the modular structure [32]. These properties are not found in the random scale-free networks of the preceding section.

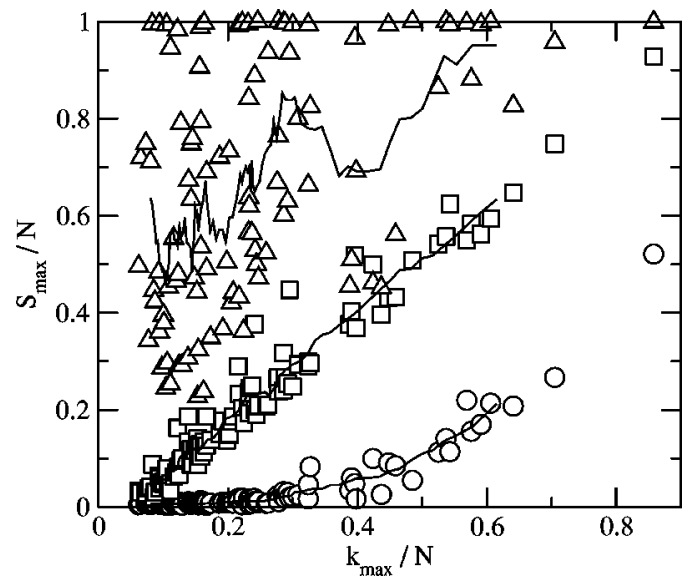

FIG. 9. Relation between the size of the largest cultural cluster and the largest degree in the network for $q=20$ (triangles), $q$ $=100$ (squares), and $q=500$ (circles). Each data point is the outcome of one realization run in a structured scale-free network of size $N=1000$. Solid lines are running averages over 10 adjacent data points for $q=20,100,500$ (top to bottom). For each value of $q$, 100 independent networks and initial conditions were generated.

As shown in Fig. 8, in the structured scale-free networks the model displays a behavior different to what we observed in random scale-free networks in the preceding section. For $q \lesssim 10$ the system settles into an ordered state. For increasing values of $q$, the order parameter undergoes a decay whose slope grows with system size. This suggests a phase transition at $q_{c} \approx 10$, in contrast to the absence of a transition point found for the randomly wired scale-free networks in the thermodynamic limit. As on large scales the structured scale-free networks have one-dimensional topology [27] it seems natural that this transition at $q_{c} \approx F$ coincides with the behavior of the model found in one-dimensional regular lattices [29].

At difference with the regular lattices, in the structured scale-free networks for $q>q_{c}$ the order parameter does not tend to zero. For the system sizes considered, the order parameter reaches a finite plateau value, indicating partial ordering of the system. Only for values $q \gg q_{c}$ the order parameter drops below the plateau value and tends to zero. This behavior may be understood by relating the size $S_{\max }$ of the largest cultural cluster with the largest degree $k_{\max }$ present in the network, as shown in Fig. 9. In the intermediate range $50<q<200$, where the plateau of the order parameter is observed, we find $S_{\max } \approx k_{\max }$ for almost all realizations. This suggests that the largest hub, the node with the largest degree, and its neighbors order such that they form the largest cluster in the absorbing state. As $q$ is reduced and its value approaches $q_{c}$ from above, the ordering goes beyond the largest hub and an increasing part of the network forms the largest cluster. On the other hand, for large values $q$ $>200$, the neighborhood of the largest hub does no longer reach complete ordering and $S_{\max }<k_{\max }$. The $q$ value for the onset of the decay of $S_{\max }$ below $k_{\max }$ is expected to be dependent on system size: as Fig. 8 shows, for increasing system size, the plateau in the order parameter extends to larger values of $q$. With increasing system size, the value of the plateau is expected to decrease as $k_{\max } / N=N^{\beta-1}$ with 
$\beta=(\gamma-1)^{-1}$, where $\gamma$ is the exponent of the degree distribution. These results suggest that in the limit $N \rightarrow \infty$, the dynamics of the social interaction model in structured networks experiences a transition similar to the one observed in a one-dimensional lattice.

\section{CONCLUSIONS}

We have found that the nonequilibrium transition between order and disorder that exists in a regular $d=2$ network for Axelrod's model of cultural influence [24] is modified by underlying complex networks with similar qualitative features that an equilibrium thermal Ising-type transition. We have shown that the transition pertains also in the presence of random long-distance connections: with increasing density of long-distance connections in a small-world network, the critical point $q_{c}(p)$ increases. Therefore, the small-world connectivity favors cultural globalization as described by the ordered state. The value of $q_{c}$ reaches a maximum for the random network obtained with a $p=1$ probability of rewiring in the small-world network construction. A transition from disorder to order is obtained increasing $p$ for a fixed value of the control parameter $q<q_{c}(p=1)$. We have also found that, for a fixed finite system size, the scale-free connectivity is more efficient than the limiting random connectivity of the small-world network in promoting the ordered state of cultural globalization. However, there is a system size scaling in the transition observed for a free scale network, so that the transition disappears in the thermodynamic limit: In the presence of scale-free interactions the order state prevails due to the presence of hubs. The consideration of structured scale free-networks restores the order-disorder transitions in spite of the hubs, but the value of the order parameter for the disordered state reveals the existence of ordered clusters.

\section{ACKNOWLEDGMENTS}

We acknowledge funding from MCyT (Spain) and FEDER (EU) through Project Nos. BFM2000-1108 and BFM2001-0341-C02-01. K.K. acknowledges a grant from CORE a/s, Denmark.
[1] J. Marro and R. Dickman, Nonequilibrium Phase Transitions in Lattice Models (Cambridge University Press, Cambridge, 1998).

[2] A.-L. Barabási and R. Albert, Rev. Mod. Phys. 74, 47 (2002).

[3] S.N. Dorogovtsev and J.F.F. Mendes, Adv. Phys. 51, 1079 (2002).

[4] D.J. Watts and S.H. Strogatz, Nature (London) 393, 440 (1998).

[5] A.-L. Barabási and R. Albert, Science 286, 509 (1999).

[6] L.A.N. Amaral, A. Scala, M. Barthélémy, and H.E. Stanley, Proc. Natl. Acad. Sci. U.S.A. 97, 11149 (2000).

[7] F. Liljeros, C.R. Edling, L.A.N. Amaral, H.E. Stanley, and Y. Ảberg, Nature (London) 411, 907 (2001).

[8] M.E.J. Newman, S. Forrest, and J. Balthrop, Phys. Rev. E 66, 035101(R) (2002).

[9] H. Ebel, L.-I. Mielsch, and S. Bornholdt, Phys. Rev. E 66, 035103(R) (2002).

[10] R. Guimera, L. Danon, A. Diaz-Guilera, F. Giralt, and A. Arenas, e-print cond-mat/0211498.

[11] A. Barrat and M. Weigt, Eur. Phys. J. B 13, 547 (2000).

[12] M. Gitterman, J. Phys. A 33, 8373 (2000).

[13] H. Hong, B.J. Kim, and M.Y. Choi, Phys. Rev. E 66, 018101 (2002).

[14] C.P. Herrero, Phys. Rev. E 65, 066110 (2002).

[15] A.D. Sánchez, J.M. López, and M.A. Rodríguez, Phys. Rev. Lett. 88, 048701 (2002).

[16] A. Aleksiejuk, J.A. Holyst, and D. Stauffer, Physica A 310, 260 (2002).
[17] A.V. Goltsev, S.N. Dorogovtsev, and J.F.F. Mendes, e-print cond-mat/0204596.

[18] S.N. Dorogovtsev, A.V. Goltsev, and J.F.F. Mendes, Phys. Rev. E 66, 016104 (2002).

[19] M. Leone, A. Vazquez, A. Vespignani, and R. Zecchina, Eur. Phys. J. B 28, 191 (2002).

[20] G. Bianconi, e-print cond-mat/0204455.

[21] M. Kuperman and G. Abramson, Phys. Rev. Lett. 86, 2909 (2001).

[22] R. Pastor-Satorras and A. Vespignani, Phys. Rev. Lett. 86, 3200 (2001).

[23] V.M. Eguíluz and K. Klemm, Phys. Rev. Lett. 89, 108701 (2002).

[24] R. Axelrod, J. Conflict Res. 41, 203 (1997); reprinted in The Complexity of Cooperation (Princeton University Press, Princeton, 1997).

[25] C. Castellano, M. Marsili, and A. Vespignani, Phys. Rev. Lett. 85, 3536 (2000).

[26] K. Klemm and V.M. Eguíluz, Phys. Rev. E 65, 036123 (2002).

[27] K. Klemm and V.M. Eguíluz, Phys. Rev. E 65, 057102 (2002).

[28] K. Klemm, V.M. Eguíluz, R. Toral, and M. San Miguel, e-print cond-mat/0205188.

[29] K. Klemm, V.M. Eguíluz, R. Toral, and M. San Miguel, e-print cond-mat/0210173.

[30] S. Milgram, Psychol. Today 2, 60 (1967).

[31] S. Wasserman and K. Faust, Social Network Analysis (Cambridge University Press, Cambridge, 1994).

[32] E. Ravasz and A.-L. Barabási, e-print cond-mat/0206130. 